\title{
Numerical Analysis of the Structural Stability of Ideal (Defect-Free) and Structurally and Morphologically Degenerated Homogeneous, Linearly- and Angle-Adjoined Nanotubes and Cylindrical Fullerenes Under Axial Loading Using Finite Element Method
}

\author{
Sadegh Imani Yengejeh* \\ Centre for Clean Environment and Energy \\ School of Environment and Science, Griffith University \\ Gold Coast Campus, Southport 4222, Australia \\ Queensland Micro- and Nanotechnology Centre, Griffith University \\ Nathan, QLD 4111, Australia \\ s.imaniyengejeh@griffith.edu.au \\ Andreas Öchsner \\ Faculty of Mechanical Engineering \\ Esslingen University of Applied Sciences \\ Esslingen 73728, Germany \\ Seyedeh Alieh Kazemi \\ Department of Mechanical Engineering, The University of Birjand \\ South Khorasan Province, Birjand, A78, 97175615, Iran \\ Maksym Rybachuk \\ School of Engineering, Griffith University \\ Southport, QLD 4222, Australia \\ Queensland Micro- and Nanotechnology Centre, Griffith University \\ Nathan, QLD 4111, Australia
}

Received 12 July 2018

Revised 16 September 2018

Accepted 24 September 2018

Published 20 November 2018

\begin{abstract}
We report on the structural stability of ideal (defect-free) and structurally and morphologically degenerate carbon nanotubes and nanotube junction systems under axial loading based on the finite element method. We estimated the values for critical buckling load for uncapped and capped single-walled carbon nanotubes (SWCNTs) and linear and angle-adjoined SWCNT heterojunctions in ideal and structurally degenerate systems containing single-, double-, triple-, pinhole- and pentagon-heptagon (i.e., $5-7$ ) structural defects and also containing a substitutional nitrogen $(\mathrm{N})$ atom inclusion under compressive loading. Absolute atomic vacancy (defect) concentration in studied SWCNTs models
\end{abstract}




\begin{abstract}
was assumed to be nil for ideal systems, and was up to 3.0 at.\% for structurally and morphologically degenerate systems. It was found that all types of structural defects and the morphological N-defect had reduced the load carrying capacity and mechanical strength in all SWCNT systems studied. The SWCNT models containing physically large vacant sites, such as triple- and pinhole-defects, displayed significantly lower critical load values compared to the systems that contained only a single-, double- or triple-vacancies. In addition, we found that capped SWCNTs performed marginally better in critical load carrying capacity compared to uncapped SWCNT systems. Furthermore, majority of the investigated structures displayed reduced load in SWCNTs with narrower tube widths, proportional to the size and the type of the defect investigated. The effects of chirality, such as zigzag- versus armchair-type, on the structural stability of the investigated SWCNT models were also investigated.
\end{abstract}

Keywords: Carbon nanostructures; finite element method; mechanical properties; critical buckling load; defects; numerical modeling.

\title{
1. Introduction
}

Following the discovery of carbon nanotubes (CNTs) Iijima, 1991, significant research effort has been expended to explore the properties and potential applications of these low-dimensional materials. Over the past two decades, it has been found that CNTs display favorable mechanical Cadek et al., 2002 and optoelectronic properties Vivien et al., 2002, high chemical inertness Qureshi et al., 2009], low-thermal degradation [Tian et al., 2004], and can be effectively doped Surivasena Livanage et al., 2014 and processed on a large scale for applied applications as structural fillers [Phang et al., 2006; Imani Yengeieh et al., 2017] or active or passive opto-electronic components [Talin et al., 2001]. In addition, studies exploring the mechanical properties and structural stability of other types of one- and twodimensional (2D) carbon nanostructures other than those of the single-walled CNTs (SWCNTs), such as fullerenes, nanocones and CNT hetero-junctions, are gradually becoming popular as these explore the potential for construction of rigid molecular templates for applied nano- and macro-molecular engineering applications Imani Yengejeh et al., 2015]. Investigations concerning the physical properties of CNTs have been formally divided into two major streams of experimental and theoretical studies. The latter employ a large variety of simulation methods including $a b$ initio quantum mechanical (QM) approaches to investigate the electronic structure (i.e., the ground state) of complex many-body molecular systems which include $\mathrm{QM} /$ molecular mechanical, density functional theory (DFT) methods and other methods to accurately model the exchange and correlation interactions. Despite offering high computational accuracy for modeling electronic properties of relatively small molecular systems, applications of QM methods to large molecular systems $(>1.0 \times 102$ atoms $)$ demand extensive computational power and long computational time Toomsalu et al., 2013]. By employing the QM approaches the mechanical properties of complex molecular systems, including those of single- and multi-walled CNT models, can be effectively estimated by analyzing force field data and the potential energy of a system [Condon and Morse, 1931; Ferguson and Kollman, 1991]. Recent studies have shown that the use of the crystal-based finite element analysis 
method (FEM) for modeling of exclusively mechanical properties of small and very large single-body and multi-body $(>1.0 \times 105$ atoms $)$ structural models is becoming popular as the FEM approach offers significantly reduced computational time and lowers the demand for resources while rendering highly plausible simulation results. Crystal-based FEM is a versatile continuum mechanics approach used for predicting mechanical properties and deformation-induced crystallographic structures, and it can be applied to solving both elastic-plastic and purely elastic problems of multi-body systems Ma et al., 2008 and complex CNT model structures Wang et al., 2009, 2016; Faria et al., 2016; Litak, 2017; Robinson and Adali, 2017; Bocko and Lengvarský,, 2017; Timesli et al., 2017; Hosseini-Hashemi and Ilkhani, 2017; Nishimura et al., 2017]. Investigation concerning the mechanical response and structural stability of these quasi-molecular systems is important, as such studies enable researchers to design and imitate the potential behavior of low-dimensional structures that bear close structural resemblance to nanostructured materials found in nature. The paragraph below presents some of the most recent findings on simulated mechanical properties of SWCNT structural systems.

Agnihotri and Basu 2010] conducted an MD simulation to investigate if a very long SWCNT can be modeled as a Kirchhoff's elastica with a small initial twist. They also indicated that the post-buckling deformation of the low-dimensional structures is strongly affected by the length, diameter and chirality of the nanostructures. Ziaee 2014] applied molecular structural mechanics to investigate the buckling properties of defective armchair and chiral SWCNTs under transverse and axial compression loading. He found that the position of the microscopic impurity in the structure of the low-dimensional material models plays a remarkable role on the change in the value of critical buckling force. Roy Chowdhury et al. 2014] performed MD simulations to study the buckling property of single- and multi-walled CNTs with different types of chirality. Their equivalent shell model gave a reasonable estimate of the critical buckling load (CBL)/strain and critical buckling torque with respect to the MD results. Wang et al. [2016] investigated the buckling response of SWCNTs under bending loads and found low- and high-strain phases in SWCNT models. The SWCNTs were simulated as thin-wall, circular, crystal systems and the governing equations have been solved by employing a continuation algorithm. Following up on these findings, Faria et al. 2016] explored the buckling properties of SWCNTs under combined bending-and-twisting loads. The study examined two chiral types of SWCNT systems, including those of armchair- and zigzag-type and of the same overall length and width. The results were presented in the form of energy diagrams and bending moments plotted against the imposed axial rotations and had shown that the armchair-type SWCNT models exhibited predominantly higher twisting-bending stiffness and strength values relative to the zigzag-type SWCNTs. Litak 2017] simulated mechanical properties of SWCNT under the influence of axial loads, and estimated the critical SWCNT diameter using two different approaches under local shell buckling mode. For the same types of SWCNT models, 
Robinson and Adali 2017] applied a nonlocal continuum mechanics approach by implementing constitutive modeling of the tubes with research results obtained for both the uniformly distributed and triangularly distributed axial loads. Bocko and Lengvarský [2017] conducted FEM studies on the buckling response of SWCNT models; they found that the structural stability of SWCNT models can be investigated by modeling these SWCNT systems as beam or shell structures and by applying an analytical shell theory. Hosseini-Hashemi and Ilkhani [2017] studied different types of structural instabilities for a spinning SWCNT models under combined loading by applying the Timoshenko and Euler-Bernoulli nonlocal beam theory Biot, 1955; Cowper, 1966 in their analysis. Their findings showed that different types of instability modes exist for simply supported SWCNT models. Based on these recent studies, it is clear that additional research efforts are needed to elucidate the effects of structural and topological defects in complex low-dimensional carbon systems, such as SWCNTs, as these systems are more likely to be found in nature.

In this study, we investigate the buckling characteristics in ideal, defect-free, homogeneous, and structurally and morphologically degenerate carbon nanostructures, namely the linearly- and angle-adjoined SWCNT and cylindrical fullerenes models under axial loading, and evaluate the impact of possible structural modifications in these models on their CBL characteristics. The investigated SWCNT and cylindrical fullerene models display significant structural aberrations, that separate them from classical low-dimensional carbon systems investigated in earlier works Imani Yengejeh et al., 2016. It is therefore conceivable that the structural stability of such asymmetric models could be greatly influenced by their axial symmetry, relative position of defects in their structure, boundary conditions and other factors. We will illustrate that the mechanical properties of such systems can be effectively studied using the research methodology presented herein.

\section{Materials and Methods}

\subsection{Spatial geometry of carbon nanostructures}

The majority of known carbon nanostructures, such as spherical and cylindrical fullerenes, linearly- and angle-joined CNT systems, and nanocones can be devised and constructed from pseudo-two-dimensional (2D) single-layer sheets of graphene, as illustrated in Fig. 1

Common homogeneous CNTs can be created by rolling a graphene sheet, and categorized as zigzag-, armchair-, or chiral- CNTs, which are defined in terms of their chiral angles, while a linearly-joined CNT molecular system is built by joining two common CNTs together along their axes of symmetry. The latter results in the introduction of Stone-Wales (5-7) defects within the immediate area of their junction. In the situation where the two connecting CNTs display the same chiral angle (i.e., zigzag, armchair, or chiral configuration), the created junction will be 


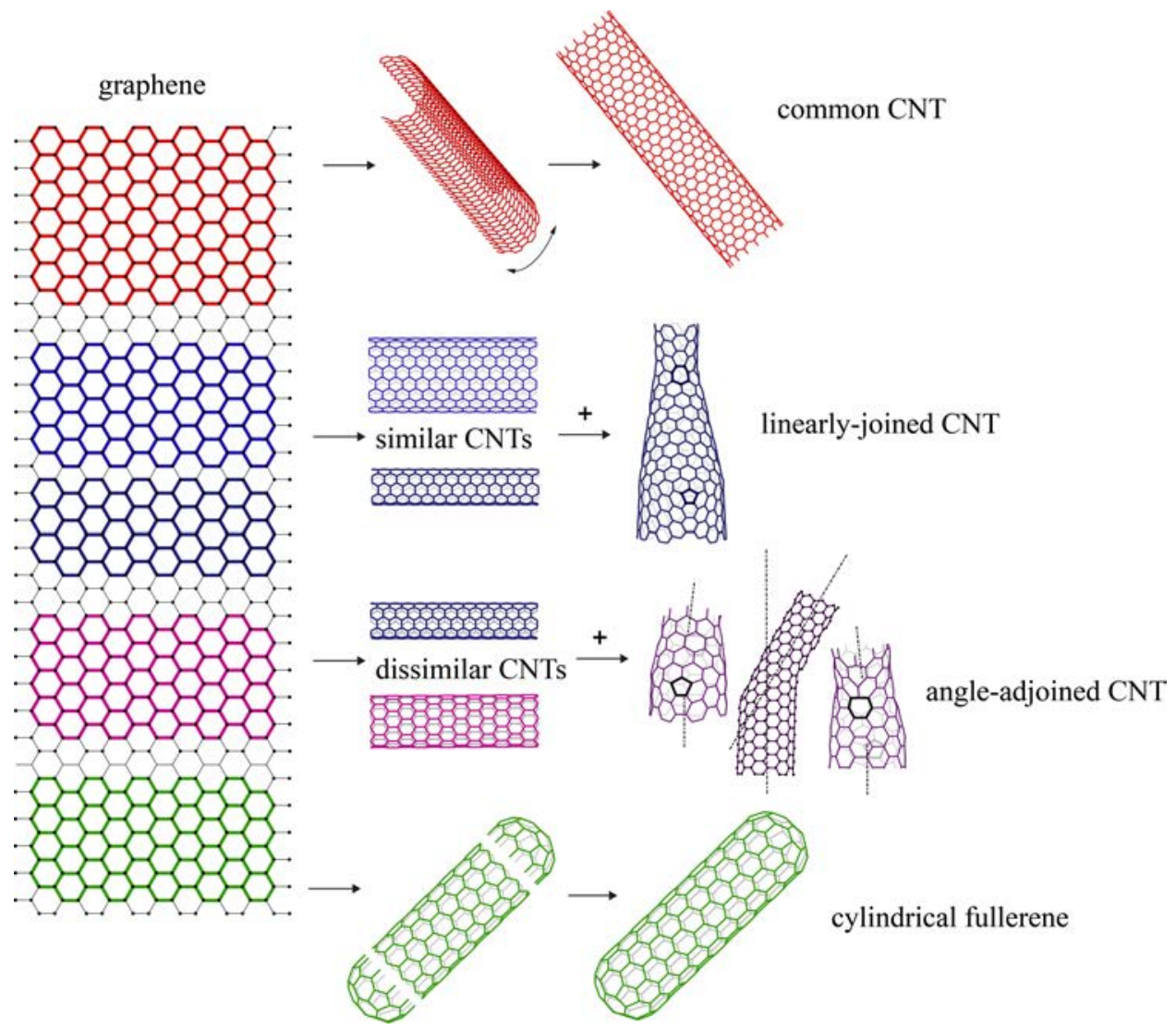

Fig. 1. Single-layer graphene as carbon precursor to common CNT, linearly- and angle-adjoined CNT and cylindrical fullerene.

straight and the 5-7 defects will appear on the same side of the junction. On the other hand, if the CNTs forming the junction are of different chiral angles, the constructed junction will have a bending angle, as illustrated in Fig. 2 .

Naturally, cylindrical fullerene structures are constructed from common CNTs those ends are capped, as shown in Fig. [3.

The aim of this study is to simulate mechanical properties of imperfect types of low-dimensional carbon models, which are more likely to be found in nature [Tran and Lambrakos, 2005: Sridhar et al., 2012: Miralles et al., 2012: Bokova-Sirosh et al., 2016]. The planned approach is to explore and predict the buckling response of common SWCNTs and their corresponding degenerated counterparts under the influence of atomic impurities as well as under different boundary conditions.

The atomic and topological defects applied to the structures of this study, are vacant sites (single-, double-, triple- and pinhole- vacancies), 5-7 pair defects, and a nitrogen $(\mathrm{N})$ substitutional defect. Nitrogen atom with its size of $65 \mathrm{pm}$ is 


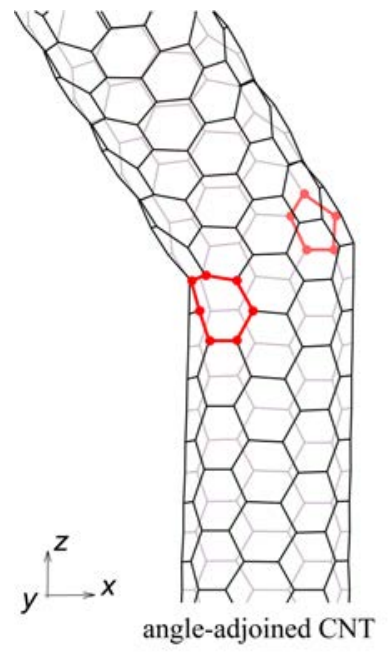

(a)

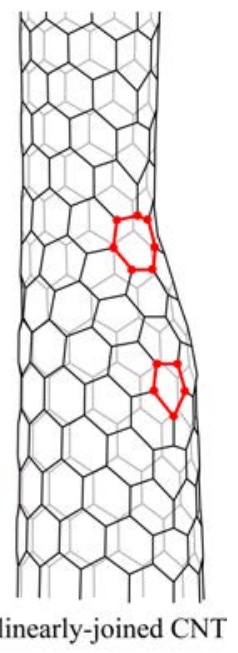

(b)

Fig. 2. Joined CNTs displaying 5-7 pair defects; (a) an angle-adjoined CNT and, (b) a linearlyjoined CNT.

armchair cylindrical fullerenes
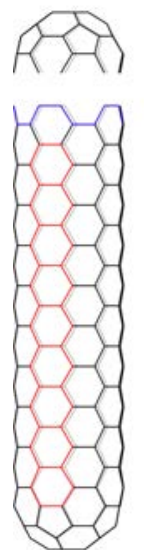

$(5-5)$
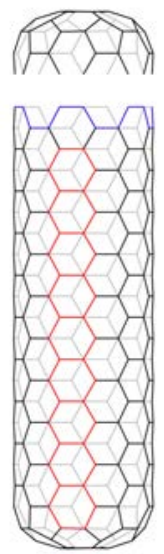

(6-6)
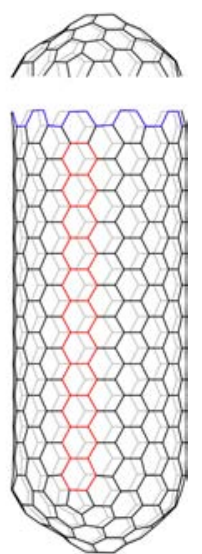

(10-10)

\section{zigzag cylindrical fullerenes}
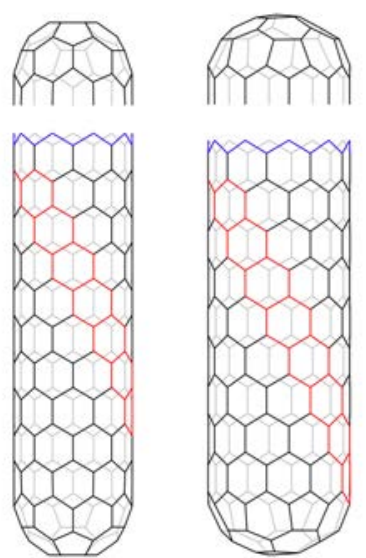

(9-0)

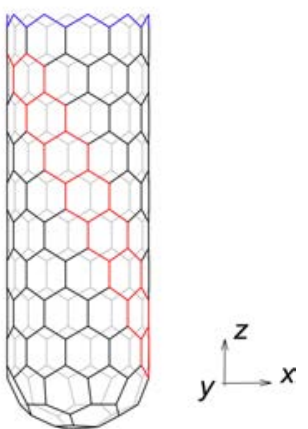

(10-0)

(a)

(b)

Fig. 3. Cylindrical fullerenes, (a) an armchair-type and, (b) a zigzag-type.

approximately $9 / 10$ of the size of a carbon atom and it is assumed to take place of a normally occupied site of carbon atom in the crystal structure of SWCNT or a fullerene model, as shown in Fig. 4 (the position of substitutional $\mathrm{N}$ atom is shown as an immobilized, ad-on attachment for clarity). 


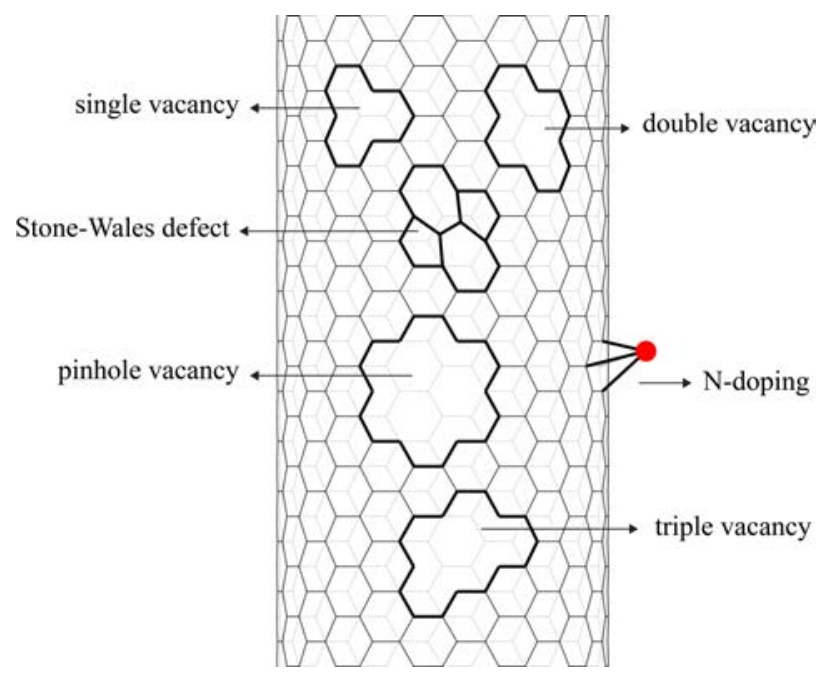

Fig. 4. Schematic representation of single-, double-, triple- and pinhole-vacancies, Stone-Wales defects and a substitution lattice (i.e., N-atom) defect in the structure of SWCNT and fullerene carbon models.

\subsection{Calculation method and experimental design}

The simulation technique performed in this study is based on the approach suggested in our earlier work $\mathrm{Li}$ and $\mathrm{Chou}, 2003$, where the low-dimensional carbon systems contain atoms and covalent bonds, with their specific lengths and bond angles are modeled as space-frame structures. When subjected to mechanical loading all of the elements bonded together in these models are assumed to be connecting loadcarrying beam members [Li and Chou, 2003; Öchsner, 2018] as shown in Fig. 5 .

We attempt to model several types of nanostructures, such as straight and angleadjoined SWCNT junctions and capped fullerenes and to investigate the CBL in these systems under different boundary conditions. The modeling procedure was the following. In step one, the spatial coordinates of atoms and their bonding vectors for all low-dimensional models were generated using Nanotube Modeler ${ }^{\circledR}$ software package (C) JCrystalSoft) and imported to a commercial FEM package Marc ${ }^{\circledR}$ (MSC Software Corporation, CA, USA), and the CBL of defect-free and ideal SWCNT models was acquired. The overall length of homogeneous and capped SWCNTs was set at $15 \mathrm{~nm}$, while the total length for straight and bending hybrid bonds was varied from 45 to $60 \mathrm{~nm}$ and from 35 to $40 \mathrm{~nm}$, respectively. The values of bond strength, $E$, for an sp2-hybridised single carbon-carbon $\mathrm{C}-\mathrm{C}, \mathrm{EC}-\mathrm{C}=5.484 \times 10^{-6} \mathrm{~N} / \mathrm{nm}^{2}$ and single carbon-nitrogen $\mathrm{C}-\mathrm{N}, \mathrm{EC}-\mathrm{N}=4.806 \times 10^{-6} \mathrm{~N} / \mathrm{nm}^{2}$ were imported to the $\mathrm{FE}$ software for processing [Song et al., 2006; Ganesan et al., 2010]. Considering the fact that the $\mathrm{C}-\mathrm{C}$ bond dissociation energy is approximately $345 \mathrm{~kJ} / \mathrm{mol}$ compared to the $\mathrm{C}-\mathrm{N}$ bond of $305 \mathrm{~kJ} / \mathrm{mol}$, the inclusion of nitrogen atom into the SWCNT lattice is a weakening factor, which overall reduces the stability of the doped structures 


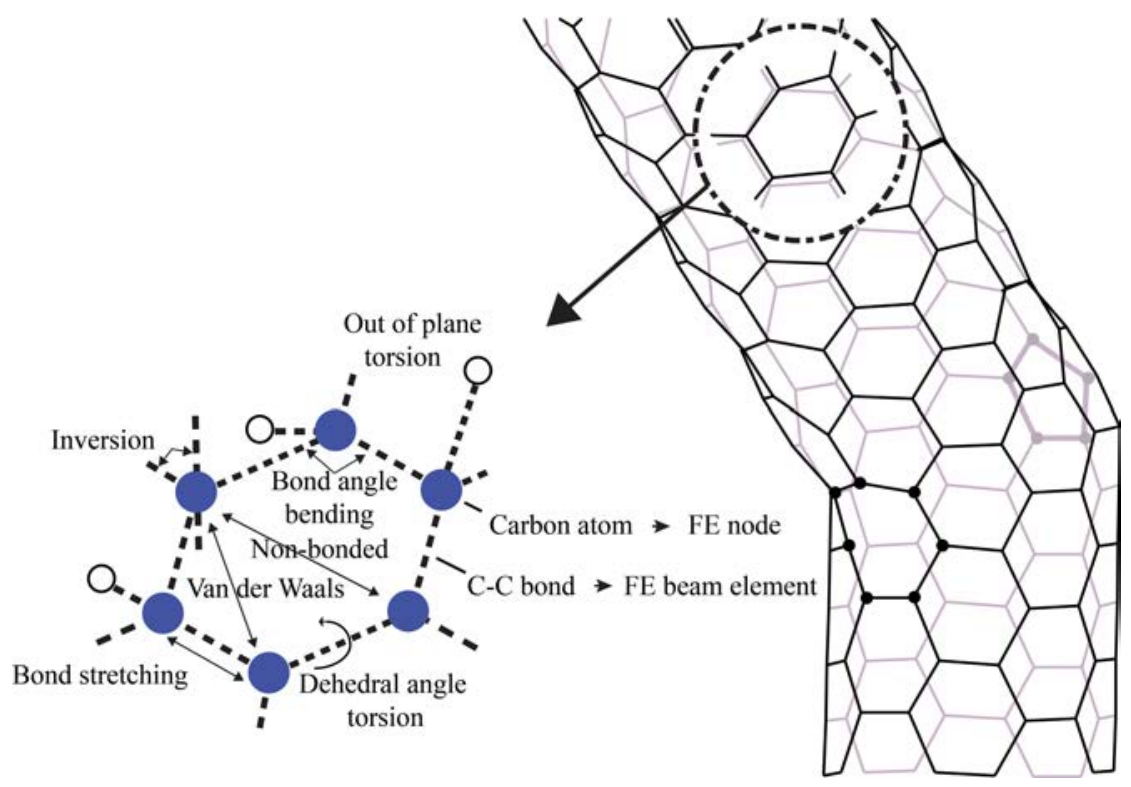

Fig. 5. Simulation of a linearly-joined SWCNT junction as a space-frame model (adopted from Lee and Lee [2012).

studied [Armentrout, 1990]. The theory of elasticity Sokolnikoff, 1956] describes the critical buckling response of a system as

$$
P_{\mathrm{cr}}=\frac{n^{2} \pi^{2} E I}{(K L)^{2}},
$$

where $P_{\text {cr }}$ is the CBL of the system, $K$ is the effective length constant, $E$ is the Young's modulus of the low-dimensional particle, $L$ is the total length of the structure, $n$ is the buckling mode and $I$ is the second moment of area. The critical buckling response of a system naturally depends on the overall geometry, boundary condition and basic configuration of the system, and during the first step, these variables are defined in the calculations. In our calculations, the values of $I$ have been empirically estimated for the ideal and degenerated SWCNT systems containing (up to 900 atoms), with $K=2$ used for the cantilever-type systems and an $n$ of 1 used for the initial simulation of the first buckling mode.

Since, homogeneous CNTs can be viewed as a hollow cylinder, therefore, the following equation can be used to define the second moment of area:

$$
I=\pi\left[(d+t)^{4}-(d+4)^{4}\right] / 64,
$$

where $d$ and $t$ are the diameter of the CNT and the thickness of the CNTs shell, respectively. Here, the $t$-values of the CNTs and their derivations are the same as the interlayer spacing of graphite $(0.34 \mathrm{~nm})$ Li and Chou, 2003; To, 2006].

In step two, some of the most common atomic defects, including vacant sites, an N-defect, and the 5-7 pair defect were applied to the ideal, defect-free SWCNT 
and fullerene models. All of the impurities were introduced and applied using a custom code in MATLAB ${ }^{\circledR}$ (MathWorks, MA, USA) to the original defect-free, ideal models at 1.0, 2.0 and 3.0 at.\% to the total number of atoms in the system.

In the final step, the CBL of degenerated SWCNT and fullerene models is obtained and compared to the nondefective ideal configurations. The comparison reference was based on the CBL of the ideal structures. The overall FEM simulation procedure is illustrated in the block diagram shown in Fig. 6]

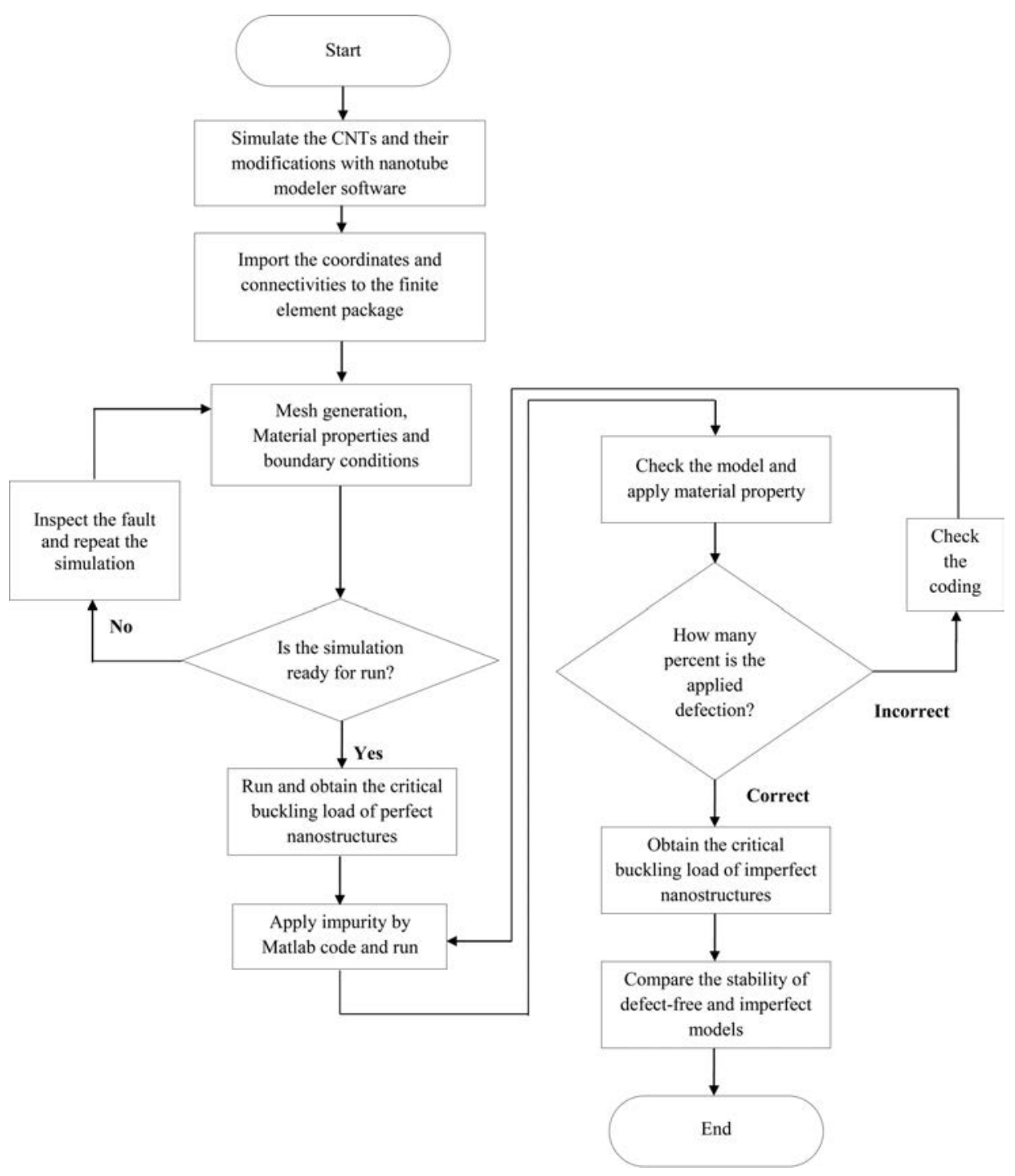

Fig. 6. Investigation procedure adopted to estimate the structural stability and CBL values in ideal and structurally degenerated SWCNT and fullerene material models. 


\section{Results and Discussion}

\subsection{Structural stability of defect-free, ideal SWCNT and fullerene structures}

The CBL of defect-free, ideal SWCNT and fullerene models under different boundary conditions is presented in Table 1 .

Generally, the FEM results demonstrate that the CBL values vary depending on the structural organization of the low-dimensional systems. More precisely, the stability of the structures depends on the physical height of the constructed models, their axial symmetry, relative position to normal reference plane and their fixation mode. The general observation is that the structural stability of the ideal SWCNT and fullerene models increases by increasing the respective diameter of the tubes.

Table 1. The values for CBL in defect-free, ideal SWCNT and fullerene models used are reference models in this study.

\begin{tabular}{|c|c|c|c|c|}
\hline Schematic view & Model & Chirality & Boundary condition & $\begin{array}{c}\text { Critical } \\
\text { buckling } \\
\text { load (nN) }\end{array}$ \\
\hline \multirow[t]{3}{*}{ 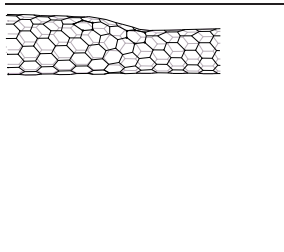 } & \multirow[t]{3}{*}{$\begin{array}{l}\text { Linearly-joined } \\
\text { CNT }\end{array}$} & $(3-3)-(5-5)$ & $\begin{array}{l}\text { Wide-end fixed } \\
\text { Narrow-end fixed }\end{array}$ & $\begin{array}{l}0.28 \\
0.13\end{array}$ \\
\hline & & $(5-5)-(10-10)$ & $\begin{array}{l}\text { Wide-end fixed } \\
\text { Narrow-end fixed }\end{array}$ & $\begin{array}{l}1.36 \\
0.5\end{array}$ \\
\hline & & $(14-14)-(16-16)$ & $\begin{array}{l}\text { Wide-end fixed } \\
\text { Narrow-end fixed }\end{array}$ & $\begin{array}{l}13.58 \\
10.73\end{array}$ \\
\hline \multirow[t]{3}{*}{000111} & \multirow[t]{3}{*}{$\begin{array}{l}\text { Angle-adjoined } \\
\text { CNT }\end{array}$} & $(3-3)-(8-0)$ & $\begin{array}{l}\text { Zigzag CNT fixed } \\
\text { Armchair CNT fixed }\end{array}$ & $\begin{array}{l}0.54 \\
0.36\end{array}$ \\
\hline & & $(5-5)-(10-0)$ & $\begin{array}{l}\text { Zigzag CNT fixed } \\
\text { Armchair CNT fixed }\end{array}$ & $\begin{array}{l}0.71 \\
0.55\end{array}$ \\
\hline & & $(7-7)-(16-0)$ & $\begin{array}{l}\text { Zigzag CNT fixed } \\
\text { Armchair CNT fixed }\end{array}$ & $\begin{array}{l}1.36 \\
1.03\end{array}$ \\
\hline \multirow{5}{*}{ 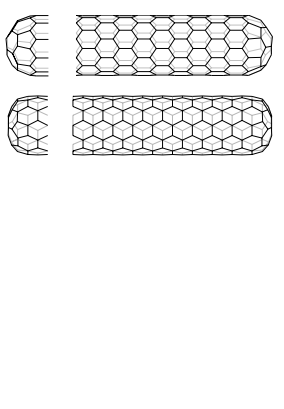 } & \multirow[t]{5}{*}{$\begin{array}{l}\text { Cylindrical } \\
\text { fullerene }\end{array}$} & (5-5) armchair CNT & $\begin{array}{l}\text { Top capped capped } \\
\text { Bottom-end fixed }\end{array}$ & $\begin{array}{l}0.57 \\
0.56\end{array}$ \\
\hline & & (6-6) armchair CNT & $\begin{array}{l}\text { Top capped capped } \\
\text { Bottom-end fixed }\end{array}$ & $\begin{array}{l}0.99 \\
0.96\end{array}$ \\
\hline & & (10-10) armchair CNT & $\begin{array}{l}\text { Top capped capped } \\
\text { Bottom-end fixed }\end{array}$ & $\begin{array}{l}4.63 \\
4.41\end{array}$ \\
\hline & & (9-0) zigzag CNT & $\begin{array}{l}\text { Top capped capped } \\
\text { Bottom-end fixed }\end{array}$ & $\begin{array}{l}0.59 \\
0.58\end{array}$ \\
\hline & & (10-0) zigzag CNT & $\begin{array}{l}\text { Top capped capped } \\
\text { Bottom-end fixed }\end{array}$ & $\begin{array}{l}0.86 \\
0.83\end{array}$ \\
\hline Sts & $\begin{array}{l}\text { Freestanding } \\
\text { CNT }\end{array}$ & $\begin{array}{l}\text { (5-5) armchair CNT } \\
\text { (6-6) armchair CNT } \\
\text { (10-10) armchair CNT } \\
\text { (9-0) zigzag CNT } \\
\text { (10-0) zigzag CNT }\end{array}$ & $\begin{array}{l}- \\
- \\
- \\
-\end{array}$ & $\begin{array}{l}0.55 \\
0.94 \\
4.31 \\
0.59 \\
0.81\end{array}$ \\
\hline
\end{tabular}




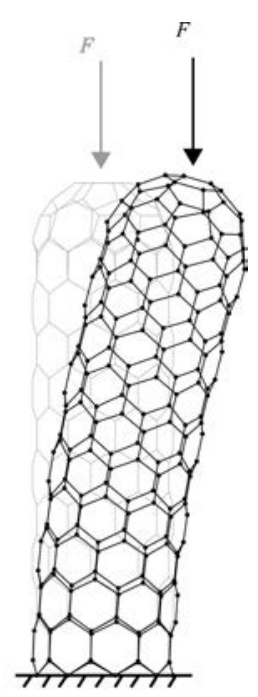

zigzag cylindrical fullerene

(a)

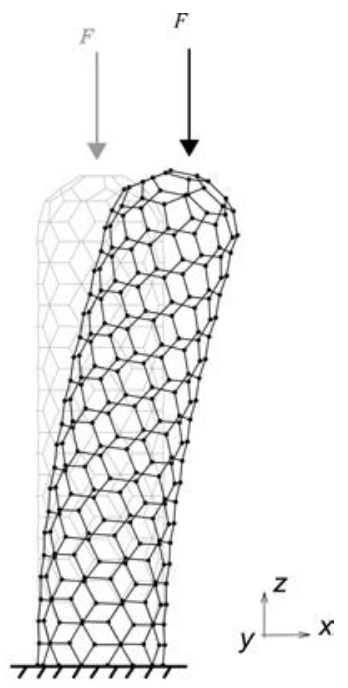

armchair cylindrical fullerene

(b)

Fig. 7. Bucking and deformation characteristics of capped and fixed SWCNTs under compressive load with (a) zigzag-type and (b) armchair-type chiral organizations.

In addition, owing to their straight configuration, linearly-joined SWCNTs display higher resistance for compression loading compared to the angle-adjoined SWCNT models.

The buckling response of capped SWCNT models with different chirality, such as the zigzag- and an armchair-types, under the compressive loading are shown in Fig. 7. Both types of SWCNTs are shown deforming under the compressive load and owing to their structural similarity display very similar buckling and deformation characteristics.

\subsection{Validation of the method}

The comparison of the FEM results and analytical values obtained from the Eq. (11) indicates the validation of the method. The CBL values of the homogeneous CNTs obtained both computationally and analytically are listed in Table 2.

The analytical results obtained from Eq. (II) provide a comparison between the accuracy of FEM analysis and the analytical calculations, with the relative difference of less than $10 \%$. Although analytical solutions are in good agreement with the computational results, it should be mentioned that the analytical calculation may not be accurate for the evaluation of the CBL in some cases, especially for CNTs with smaller diameters, since it assumes the CNTs as hollow cylinders. However, the nanomaterials are considered here as cylinder-shaped trusses. Therefore, we also compared our FE outcome to the ones evaluated by other numerical approaches. 
Table 2. The values of CBL obtained from FEM and analytical equation.

\begin{tabular}{|c|c|c|c|c|c|c|c|}
\hline \multirow[t]{2}{*}{ CNT type } & \multirow[t]{2}{*}{$\begin{array}{l}\text { chirality } \\
(\mathrm{n}, \mathrm{m})\end{array}$} & \multirow[t]{2}{*}{$\begin{array}{l}\text { diameter } \\
(\mathrm{nm})\end{array}$} & \multirow[t]{2}{*}{$\begin{array}{l}\text { Length } \\
(\mathrm{nm})\end{array}$} & \multirow{2}{*}{$\begin{array}{l}\text { Young's } \\
\text { modulus } \\
(\mathrm{TPa})\end{array}$} & \multicolumn{2}{|c|}{$\begin{array}{c}\text { Critical buckling } \\
\text { load }(\mathrm{nN})\end{array}$} & \multirow[t]{2}{*}{$\begin{array}{c}\text { Relative } \\
\text { difference in\% }\end{array}$} \\
\hline & & & & & $\begin{array}{l}\text { FEM } \\
\text { results }\end{array}$ & $\begin{array}{l}\text { Analytical } \\
\text { solution }\end{array}$ & \\
\hline armchair & $(5-5)$ & 0.673 & 15 & 1.042 & 0.549 & 0.595 & 7.5 \\
\hline armchair & $(6-6)$ & 0.808 & 15 & 1.042 & 0.907 & 0.931 & 2.58 \\
\hline armchair & $(10-10)$ & 1.346 & 15 & 1.042 & 4.312 & 4.032 & 6.9 \\
\hline zigzag & $(9-0)$ & 0.699 & 15.4 & 1.032 & 0.588 & 0.643 & 8.5 \\
\hline zigzag & $(10-0)$ & 0.793 & 15.4 & 1.035 & 0.809 & 0.782 & 3.45 \\
\hline
\end{tabular}

Our results are to a great extent comparable to the ones obtained bv Bocko and Lengvarský [2017] where they applied a shell beam theory and obtained the CBL of an armchair $(6,6)$ and a zigzag $(10,0) \mathrm{CNT}$ in a very close range compared to our results (with the length of $15 \mathrm{~nm}$ ).

In addition, our results are also verified with those evaluated by Ansari et al. [2011], where MD simulations of nanotubes with chirality types of $(8,8)$ and $(14,0)$, respectively, were realized. They incorporated Eringen's nonlocality into the shell theory in order to include the small-scale effects on the axial buckling of SWCNTs with arbitrary boundary conditions. Furthermore, Poelma et al. 2012] applied the same MD simulation to evaluate the CBL of different CNTs versus the CNT length to diameter ratio. Within their MD simulation, they applied a spring force identify in the boundary conditions and extracted the reaction forces during the MD simulation. They examined the buckling property of a CNT with the diameter of $0.783 \mathrm{~nm}$, and found the CBL of the CNT with various lengths. Their value of CBL of the CNT with similar aspect ratio was comparable to ours with the relative difference under 10\%. Comparing all mentioned numerical simulations, it can be noted that the FE approach is quite reliable compared to MD simulations while the latter one is more restricted to smaller models and is very demanding in regards to computational resources.

\subsection{Vertical instability of structurally degenerated $S W C N T$ and fullerene models}

In our work, the effects of introducing four different types of vacant sites, a StoneWaals defect and, an N-substitutional defect, as shown in Fig. 4. at 1.0, 2.0 and 3.0 at.\% to the total of carbon atoms in the SWCNT and fullerene models were investigated. Figures 813 illustrate that the presence of any type of a vacancy or defect in the structure of the model reduces the values of CBL for all nanostructures models. Furthermore, these figures reveal that all types of structural defects reduce the structural stability of the models. In the case for the angle-adjoined SWCNT junction systems, the change in the CBL values for fixed zigzag-type SWCNT was found to be higher when compared to the armchair-type chiral SWCNT system. 
Notably, at 3.0 at.\% (i.e., the highest defect concentration), the CBL values of (77)-(16-0) angle-adjoined SWCNT in the zigzag-fix boundary condition were found to be reduced by nearly $5.0 \%$, whereas the reduction for the similar fixed SWCNT model with armchair-type chirality was found to be just over $4.0 \%$. The buckling characteristics of linearly-aligned fixed SWCNT junctions, those of wider and narrower diameters, were found comparable with the wider tubes displaying marginally higher structural stability. For instance, in the case of $(8,8)-(15,15)$ linearly-joined SWCNT hybrid system fixed at the wide end, the calculated reduction in the CBL was observed to be nearly $10.0 \%$; this value was lower than the one that was found for the same type of SWCNT hybrid system with fixed at the narrow end.

Comparing the data in Fig. 8 for the linear- and angle-adjoined SWCNTs, it is noted that apart from the tube diameter which directly influences the structural stability of the models, the linearly-joined SWCNTs display higher resistance compared to their angle-adjoined counterparts. Furthermore, the overall formation of junction region of these hybrid systems has a significant impact on their structural stability. More specifically, it is indicated that the structural strength of the systems with parallel longitudinal axes is greater than the ones possessing bending junctions.

The reduction in the structural stability of the double vacancy defected models (all configurations) was higher compared to the SWCNT models containing single vacancy defect. A closer look at Fig. 9] it is indicated that SWCNT with wider diameters are significantly affected by introducing double vacancy defects to their configurations in comparison with the CNT-related systems possessing narrower diameters. Comparing the structural stability of common SWCNTs and cylindrical fullerene (both structures), the highest reduction in the CBL values was observed for the SWCNT systems containing the $(10,10)$ armchair-type chirality, followed by SWCNTs with a zigzag-type chiral organization. The least affected models were SWCNT systems containing the $(9,0)$ and then $(5,5)$ SWCNTs.

According to Fig. 10 the difference between the simulated CBL values of the ideal and triple vacancy defected hybrids was significant, particularly in the case of SWCNTs hybrids containing $(7,7)-(16,0)$ type defects, in which the CBL was recorded at approximately $50 \%$ corresponding to 3.0 at. $\%$ of triple vacancy defect. While the structural stability of wider hybrid SWCNT systems, such as $(7,7)-(16,0)$ and $(8,8)-(16,16)$ reduced markedly, the thinner two-junction SWCNT systems were found unaffected by these types of structural defects.

As shown in Fig. 11, the pinhole vacancy defect had the most significant impact on the structural stability of the material models, including linearly- and angleadjoined CNTs, homogenous SWCNTs and cylindrical fullerenes. Such impurity in the structures caused up to a $70 \%$ reduction in the CBL in some circumstances. More precisely, in the case of linearly-joined CNTs, the reduction in the CBL of the system with narrow-end fixed was comparatively larger than the one for the systems with wide-end fixed boundary condition. In addition, by increasing the 


\section{Defect concentration (at. \%)}

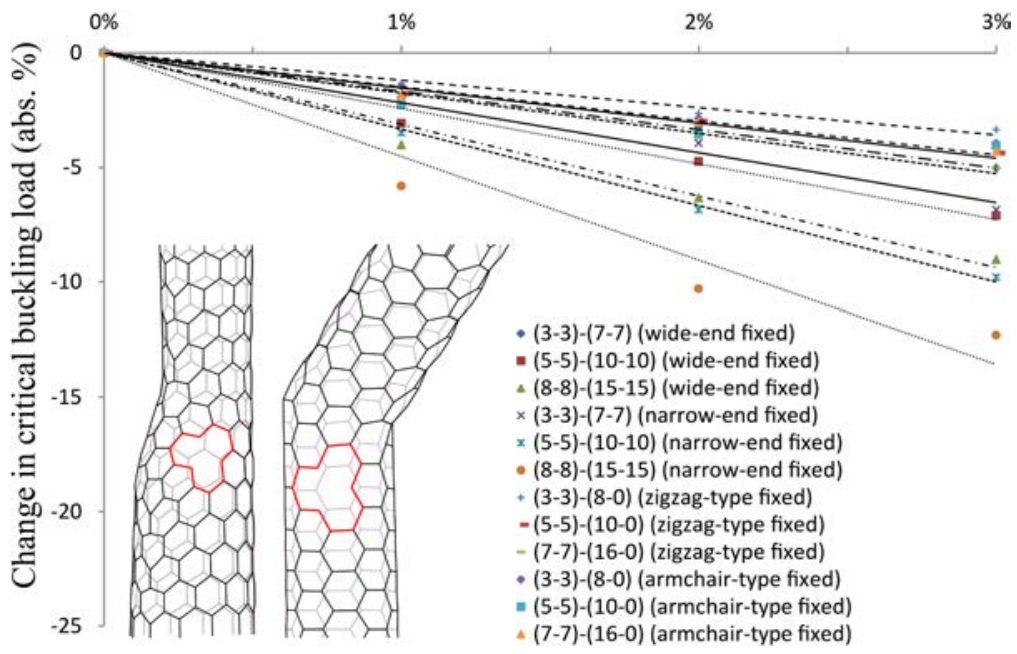

(a)

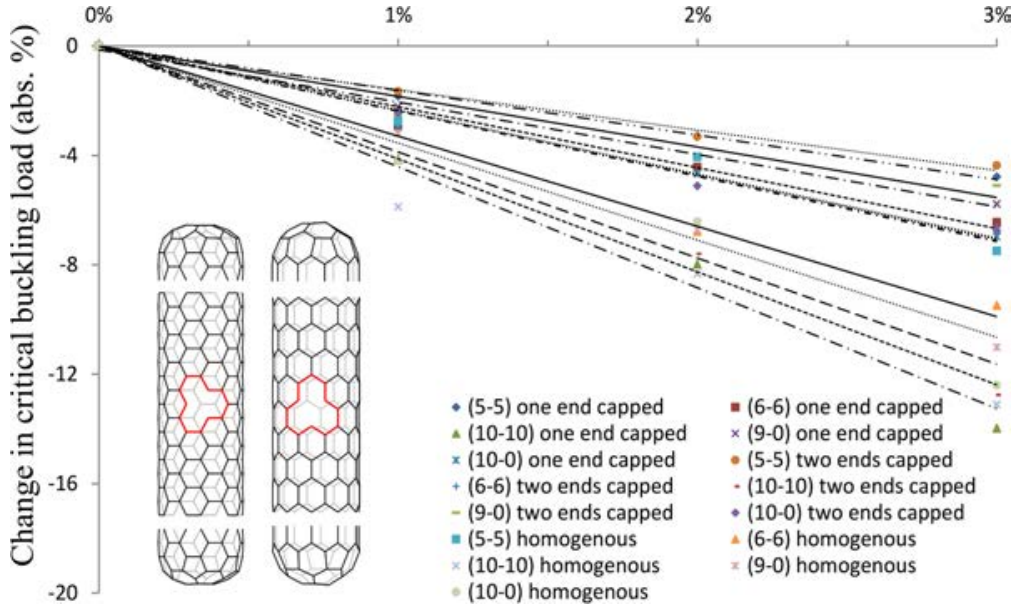

(b)

Fig. 8. Change in the CBL values (abs.\%) for (a) degenerated linearly- and angle-adjoined SWCNT hybrid systems with single vacancy defects and fixed armchair- and zigzag-type organizations and (b) degenerated homogeneous SWCNTs and cylindrical fullerenes under axial compressive load.

CNT diameter, the values of CBL of the CNT-related hybrids reduces markedly. The overall response of homogeneous CNTs and cylindrical fullerenes was comparatively similar, apart from the models containing wider diameters, e.g., (10-10) armchair CNT, where the two-end capped cylindrical fullerene had a slight reduction in the value of CBL compared to the other configurations with similar chirality. 


\section{Defect concentration (at. \%)}

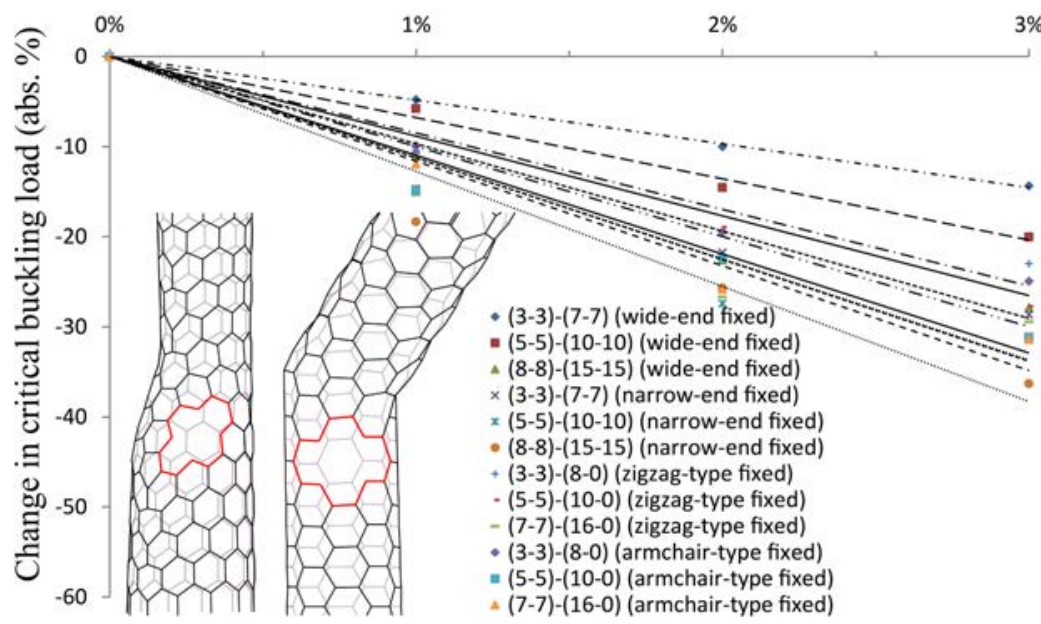

(a)

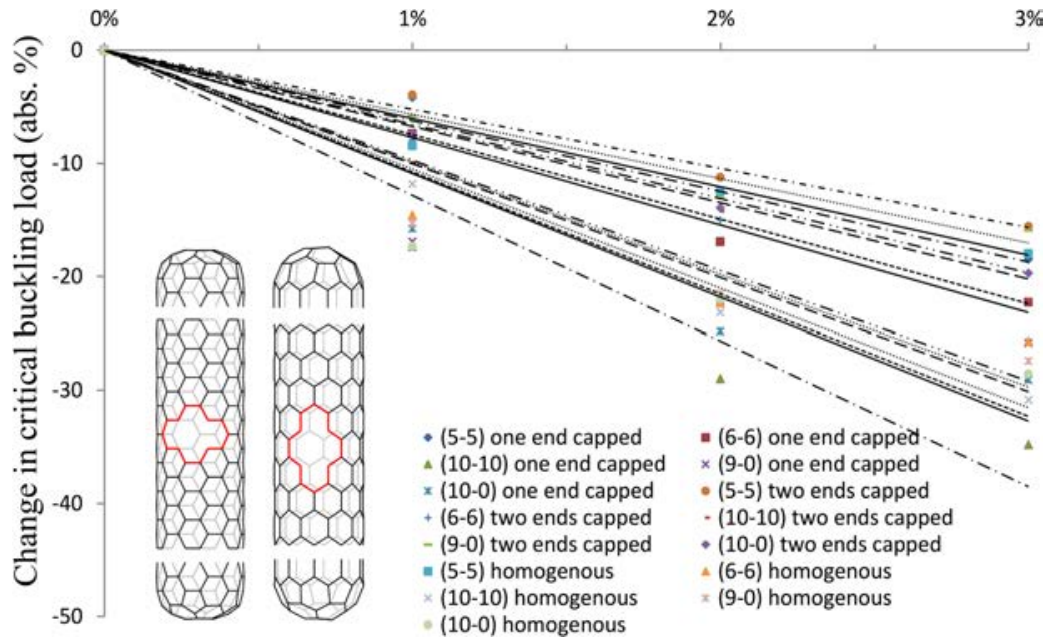

(b)

Fig. 9. Change in the CBL (in abs.\%) for (a) degenerated linearly- and angle-adjoined SWCNT hybrid systems with double vacancy defects and fixed armchair- and zigzag-type organizations and (b) degenerated homogeneous SWCNTs and cylindrical fullerenes under axial compressive load.

Introducing the 5-7 pair defect in the low-dimensional configurations of SWCNT was found to have a minor influence on the buckling load of these structures when compared to the vacant sites, as illustrated in Fig. 12. Overall, the change in the CBL for all models did not exceed $10.0 \%$. With regards to nondefective configurations. The maximum change in the CBL values was found in the linearly-joined SWCNT 


\section{Defect concentration (at. \%)}

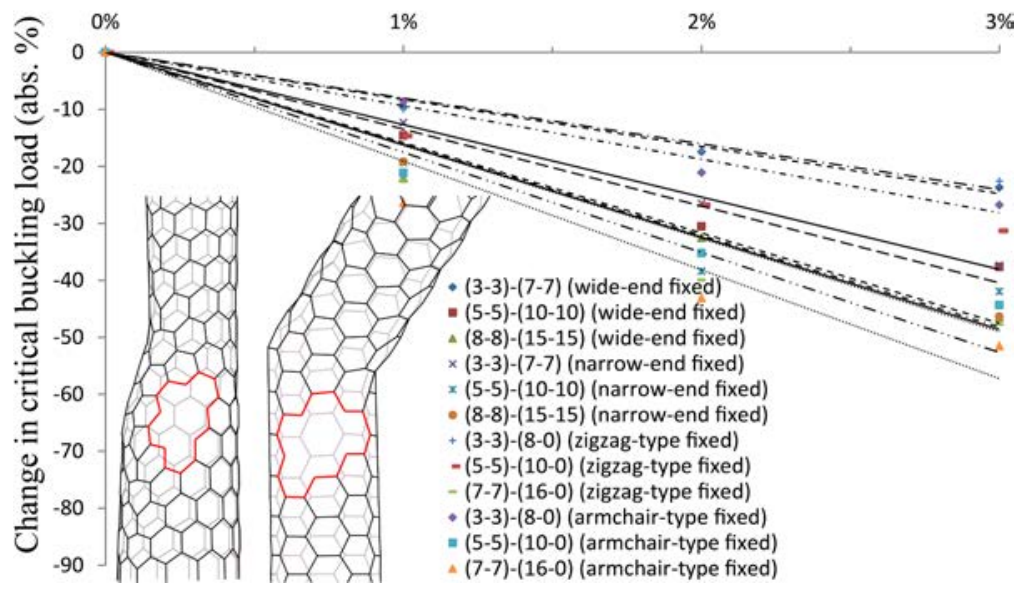

(a)

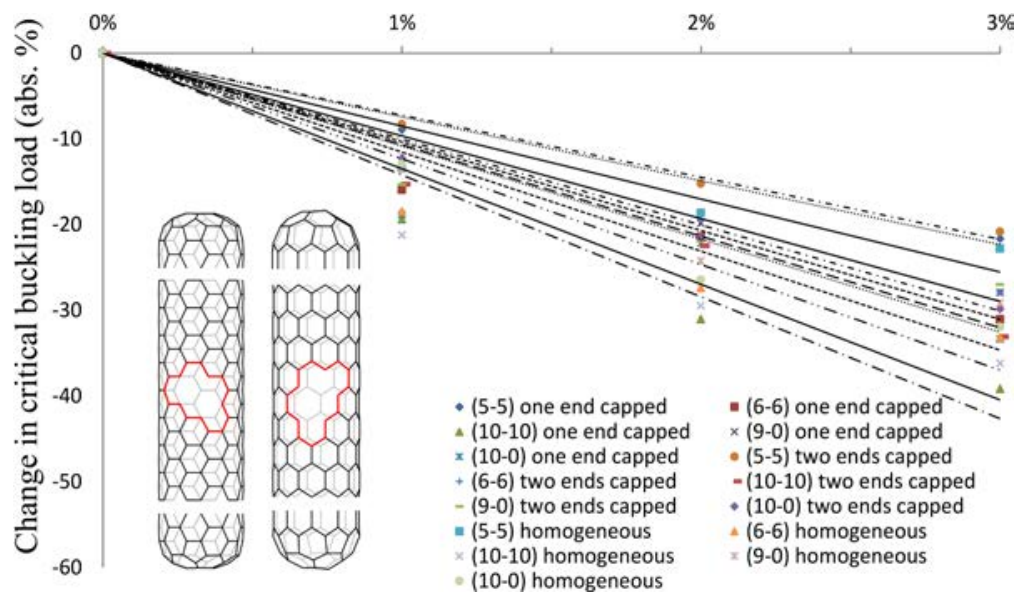

(b)

Fig. 10. Change in the CBL (in abs.\%) for (a) degenerated linearly- and angle-adjoined SWCNT hybrid systems with triple vacancy defects and fixed armchair- and zigzag-type organizations and (b) degenerated homogeneous SWCNTs and cylindrical fullerenes under axial compressive load.

hybrid systems containing $(8,8)-(16,16)$ chirality owing narrow-end fixed boundary condition, by approximately $9.3 \%$.

Followed by 5-7 pair defect, N-doping had the least impact on the buckling response of the low-dimensional structures, as shown in Fig. 13. More specifically, no change was observed in the SWCNT hybrid systems of more than 10.0\%, apart from the SWCNT hybrid system containing the $(8,8)-(15,15)$ chirality; the latter was found to contain almost $11.0 \%$. 


\section{Defect concentration (at. \%)}

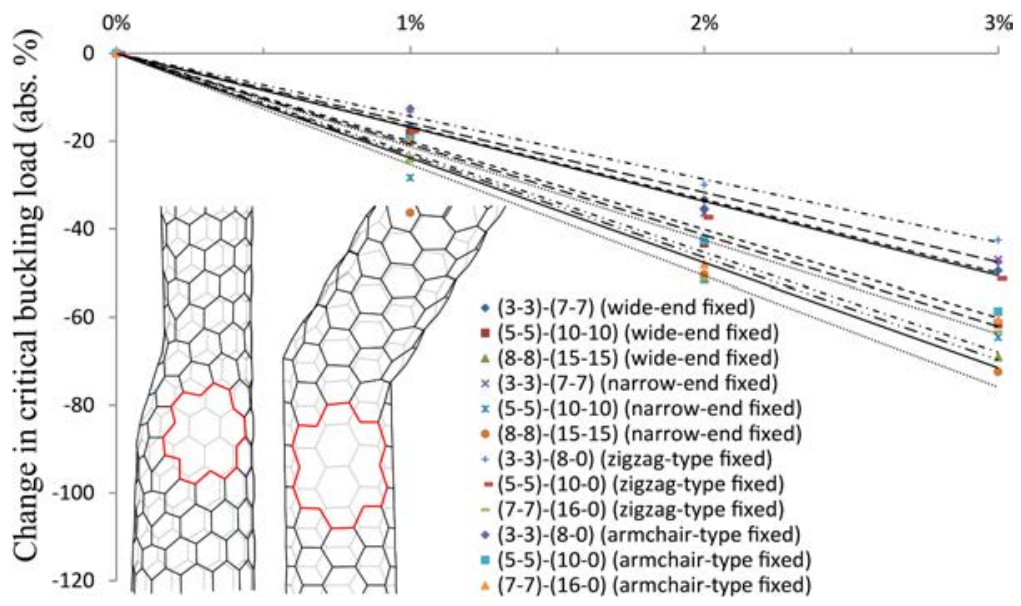

(a)

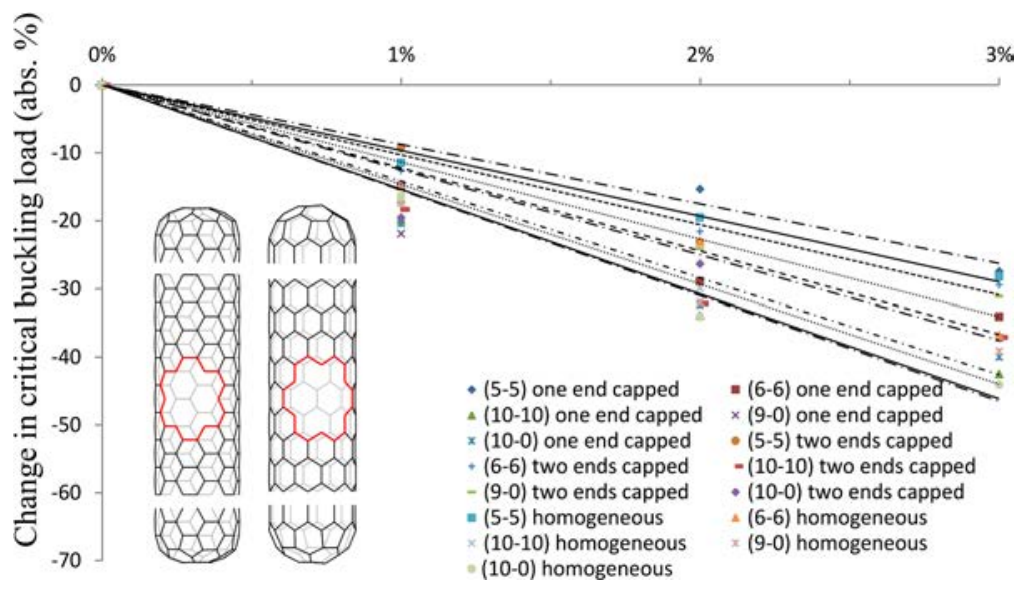

(b)

Fig. 11. Change in the CBL (in abs.\%) for (a) degenerated linearly- and angle-adjoined SWCNT hybrid systems with pinhole vacancy defects and fixed armchair- and zigzag-type organizations and (b) degenerated homogeneous SWCNTs and cylindrical fullerenes under axial compressive load.

The results indicate that the change in the CBL values of material models depends on the type and quantity of the structural defects. More specifically, it is noted that Stone-Wales and substituting dopants defects have minor influence on the structural stability of the models in comparison with the carbon vacancy defects (particularly, pinhole vacancy), which reduce the CBL of the nanostructures up to more than $60 \%$. As previously predicted, it was observed that the CBL of vacancy defective structures reduces as the size of the defect increase. More precisely, the 


\section{Defect concentration (at. \%)}

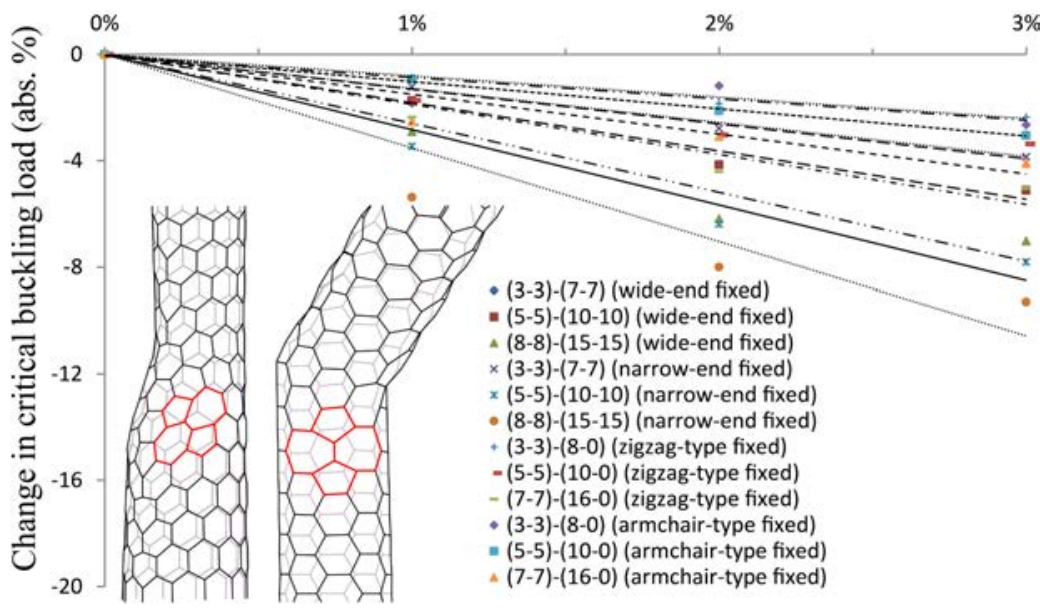

(a)

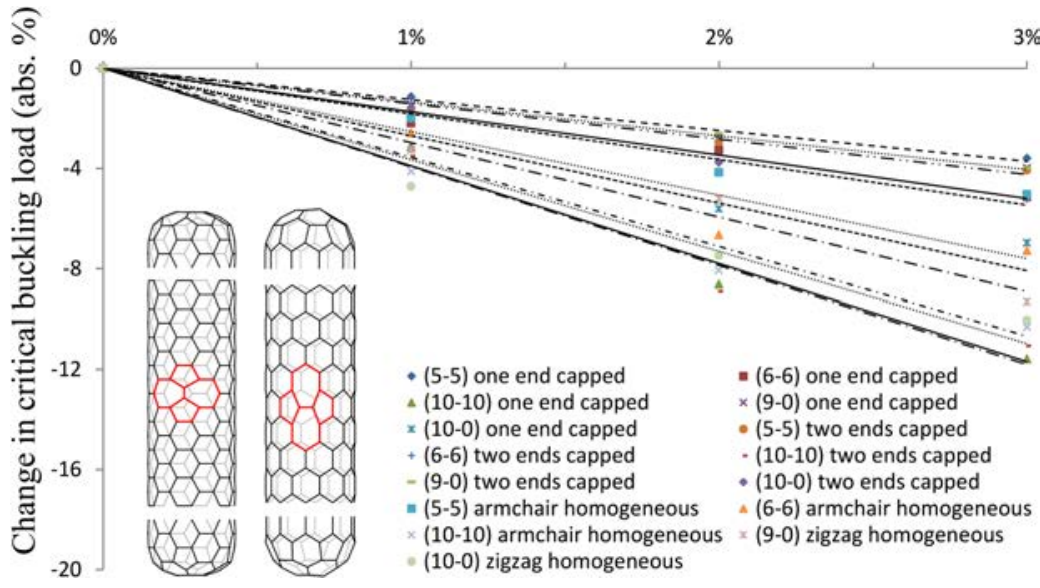

(b)

Fig. 12. Change in the CBL (in abs.\%) for (a) degenerated linearly- and angle-adjoined SWCNT hybrid systems with 5-7 pair defects and fixed armchair- and zigzag-type organizations and (b) degenerated homogeneous SWCNTs and cylindrical fullerenes under axial compressive load.

difference between the change in the CBL of the single-vacancy and pinhole vacancy defective linearly-joined CNTs is up to $50 \%$ in the case of $(8,8)-(15,15)$. It can be drawn from the computational results that structural modifications could have a significant impact on the mechanical properties of all types of nanostructures including joined CNTs, homogeneous CNTs and cylindrical fullerenes. Such influence leads to a remarkable change in the $\mathrm{CBL}$, and as a consequence, reduction in the structural stability of the low-dimensional material models. 


\section{Defect concentration (at. \%)}

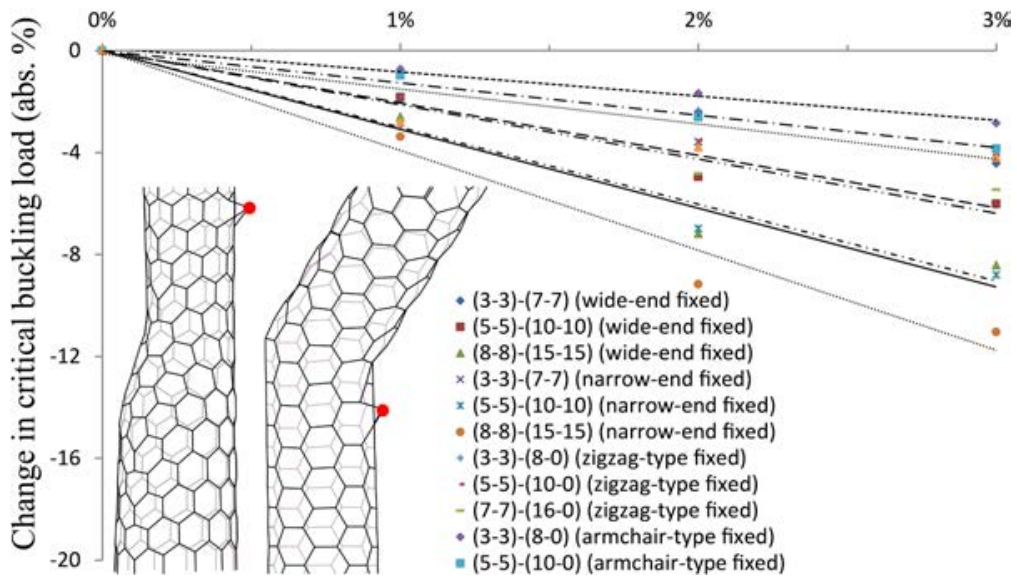

(a)

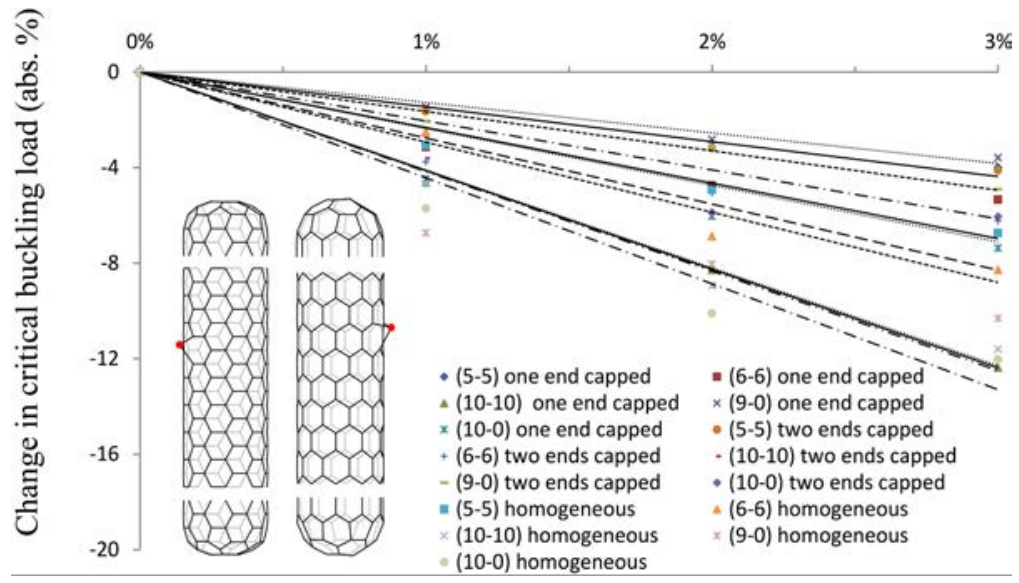

(b)

Fig. 13. Change in the CBL in abs.\% for N-doped (a) degenerated straight and knee SWCNT hybrid junctions with different chirality and boundary conditions and (b) homogeneous and capped SWCNT under axial compressive load.

\section{Summary and Conclusions}

Several types of carbon nanostructures including homogeneous, linearly- and angleadjoined SWCNTs, and cylindrical fullerenes (in both one end capped and two end capped structures) were simulated and their structural stability was investigated through applying a computational approach. Due to the diversity of the investigated material models, an attempt was made to categorize the low-dimensional structures based on their shape and configurations. Following this idea, homogeneous CNTs and cylindrical fullerenes were examined in a same category and 
linearly- and angle-adjoined were investigated as they are both hybrid nanostructures. One of the most highlighted features of this study was the application of different CNT models with variable charalities, as well as boundary conditions. Several material models with different chirality and diameters were examined in order to evaluate the mechanical properties of the degenerated structures. The results indicate that all models of degenerated carbon nano-material models have their own specific critical properties and this is completely dependent on the structural configuration of the low-dimensional structures. More specifically, linearly-joined CNT models display higher stability to external forces in comparison with their angleadjoined counterparts, due to their straight configurations. In addition, based on the obtained results of homogeneous CNTs and cylindrical fullerenes, it was indicated that these structures display a comparatively similar response to reduction in the CBL, since their overall configurations are similar at large extent. It was also noted that the CBL of nano-material models reduces by introducing microscopic impurities. This reduction leads to lower structural stability of the material models depending on the type and the quantity of the applied defects. These defects were listed as vacancy defects (single-, double-, triple- and pinhole vacancies), substitution dopant $(N)$, and Stone-Wales pair defects. The computational results showed that pinhole vacancy and Stone-Wales pair defects had the most and the least influence on the structural strength of carbon nanostructures, respectively. It was also revealed that the boundary condition had a significant impact on the evaluation of the buckling properties of asymmetric models. For instance, in the case of linearly-joined CNT structures, the change in the CBL of the CNT hybrids in the wide-end fixed was comparatively larger than the one with the narrow-end fixed boundary condition. Similarly to linear CNT hybrids, angle-adjoined CNTs with armchair-type fixed display larger change in the CBL in comparison with those with zigzag-type fixed boundary condition. It was discussed that the buckling properties of structurally identical configurations (such as homogeneous CNTs and cylindrical fullerenes) are comparatively similar. This is probably because that the mechanical properties of the CNT structures are strongly dependent on the shape and configuration of the low-dimensional material models. With regards to the structural defects, it was indicated that the change in the CBL of models with vacancy defect (in its single form) and N-doped conditions is comparatively similar and had a relatively lower influence on the CBL. In contrast, triple and pinhole vacancies had a significant impact on the CBL of the material models and reduce the structural stability of the low-dimensional structures at the largest degree. Apart from the investigation of the mechanical properties of the CNT material models based on FEM and its particular restrictions, other mechanical approaches such as QM and DFT techniques are strongly advised to evaluate the properties of the nanostructures in a more nonrestricted condition, which is currently being investigated by the authors. 


\section{References}

Agnihotri, P. K. and Basu, S. [2010] "Single-walled nanotubes as kirchhoff elasticas," International Journal of Applied Mechanics 2(04), 719-743.

Armentrout, P. B. [1990] "Bonding energetics in organometallic Compounds," Periodic Trends in Transition Metal Bonds to Hydrogen, Carbon, and Nitrogen, Vol. 428, American Chemical Society, pp. 18-33.

Biot, M. A. [1955] "Theory of elasticity and consolidation for a porous anisotropic solid," Journal of Applied Physics 26, 182-185.

Bocko, J. and Lengvarský, P. [2017] "Buckling of single-walled carbon nanotubes with and without defects," Journal of Mechanical Science and Technology 31, 1825-1833.

Bokova-Sirosh, S. N., Kuznetsov, V. L., Romanenko, A. I., Kazakova, M. A., Krasnikov, D. V., Tkachev, E. N., Yuzyuk, Y. I. and Obraztsova, E. D. [2016] "Investigation of defectiveness of multiwalled carbon nanotubes produced with $\mathrm{Fe}$-Co catalysts of different composition," Journal of Nanophotonics 10(1), 012526.

Cadek, M., Coleman, J. N., Barron, V., Hedicke, K. and Blau, W. J. [2002] "Morphological and mechanical properties of carbon-nanotube-reinforced semicrystalline and amorphous polymer composites," Applied Physics Letters 81, 5123-5125.

Condon, E. U. and Morse P. M. [1931] "Quantum mechanics of collision processes I. Scattering of particles in a definite force field," Reviews of Modern Physics 3, 43.

Cowper, G. R. [1966] "The shear coefficient in Timoshenko's beam theory," Journal of Applied Mechanics 33, 335-340.

Faria, B., Silvestre, N. and Lopes, J. N. C. [2016] "Mechanical behaviour of carbon nanotubes under combined twisting-bending," Mechanics Research Communications 73, 19-24.

Ferguson, D. M. and Kollman, P. A. [1991] "Can the Lennard-Jones 6-12 function replace the 10-12 form in molecular mechanics calculations?" Journal of Computational Chemistry 12, 620-626.

Ganesan, Y., Peng, C., Lu, Y., Ci, L., Srivastava, A., Ajayan, P. M. and Lou, J. [2010] "Effect of nitrogen doping on the mechanical properties of carbon nanotubes," ACS Nano 4, 7637-7643.

Hosseini-Hashemi, S. and Ilkhani, M. R. [2017] "Nonlocal modeling for dynamic stability of spinning nanotube under axial load," Meccanica 52, 1107-1121.

Iijima, S. [1991] "Helical microtubules of graphitic carbon," Nature 354, 56-58.

Imani Yengejeh, S., Akbar Zadeh, M. and Öchsner, A. [2015] "On the tensile behavior of hetero-junction carbon nanotubes," Composites Part B 75, 274-280.

Imani Yengejeh, S., Kazemi, S. A. and Öchsner, A. [2016] "Advances in mechanical analysis of structurally and atomically modified carbon nanotubes and degenerated nanostructures: A review," Composites Part B: Engineering 86, 95-107.

Imani Yengejeh, S., Kazemi, S. A. and Öchsner, A. [2017] "Carbon nanotubes as reinforcement in composites: A review of the analytical, numerical and experimental approaches," Computational Materials Science 136, 85-101.

Lee, J. H. and Lee, B. S. [2012] "Modal analysis of carbon nanotubes and nanocones using FEM," Computational Materials Science 51, 30-42.

Li, C. and Chou, T. W. [2003] "A structural mechanics approach for the analysis of carbon nanotubes," International Journal of Solids and Structures 40, 2487-2499.

Litak, G. [2017] "Buckling behaviour of single-walled carbon nanotubes under axial loading," Advances in Science and Technology 11(1), 208-211.

Ma, D., Friák, M., Neugebauer, J., Raabe, D. and Roters, F. [2008] "Multiscale simulation of polycrystal mechanics of textured $\beta$-Ti alloys using ab initio and crystal-based finite element methods," Physica Status Solidi (b) 245, 2642-2648. 
Miralles, P., Johnson, E., Church, T. L. and Harris, A. T. [2012] "Multiwalled carbon nanotubes in alfalfa and wheat: Toxicology and uptake," Journal of the Royal Society Interface $\mathbf{9}(77)$, 3514-3527.

Nishimura, M., Takahashi, N. and Takagi, Y. [2017] "Relationship between local buckling and atomic elastic stiffness in multi-walled carbon nanotubes under compression and bending deformations," Computational Materials Science 130, 214-221.

Öchsner, A. [2018] Computational Statics and Dynamics: An Introduction Based on the Finite Element Method (Springer, Singapore).

Phang, I. Y., Ma, J., Shen, L., Liu, T. and Zhang, W. D. [2006] "Crystallization and melting behavior of multi-walled carbon nanotube-reinforced nylon-6 composites," Polymer International 55, 71-79.

Poelma, R. H., Sadeghian, H., Koh, S. and Zhang, G. Q. [2012] "Effects of single vacancy defect position on the stability of carbon nanotubes," Microelectronics Reliability 52, 1279-1284.

Qureshi, A., Kang, W. P., Davidson, J. L. and Gurbuz, Y. [2009] "Review on carbonderived, solid-state, micro and nano sensors for electrochemical sensing applications," Diamond and Related Materials 18, 1401-1420.

Robinson, M. T. A. and Adali, S. [2017] "Buckling of nonuniform carbon nanotubes under concentrated and distributed axial loads," Mechanical Sciences 8, 299-305.

Roy Chowdhury, A. N., Wang, C. M. and Koh, S. J. A. [2014] "Continuum shell model for buckling of armchair carbon nanotubes under compression or torsion," International Journal of Applied Mechanics 6(01), 1450006.

Sokolnikoff, I. S. [1956] Mathematical Theory of Elasticity (McGraw-Hill Book Company).

Song, H. Y., Sun, H. M. and Zhang, G. X. [2006] "Molecular dynamic study of effects of Si-doping upon structure and mechanical properties of carbon nanotubes," Communications in Theoretical Physics 45, 741-744.

Sridhar, V., Kim, H. J., Jung, J. H., Lee, C., Park, S. and Oh, I. K. [2012] "Defectengineered three-dimensional graphene-nanotube-palladium nanostructures with ultrahigh capacitance," ACS Nano 6(12), 10562-10570.

Suriyasena Liyanage, L., Xu, X., Pitner, G., Bao, Z. and Wong, H. S. P. [2014] "VLSIcompatible carbon nanotube doping technique with low work-function metal oxides," Nano Letters 14, 1884-1890.

Talin, A. A., Dean, K. A. and Jaskie, J. E. [2001] "Field emission displays: A critical review," Solid-State Electronics 45, 963-976.

Tian, Y. J., Zhang Y. L., Yü, Q., Wang, X. Z., Hu, Z., Zhang Y. F. and Xie, K. C. [2004] "Effect of catalysis on coal to nanotube in thermal plasma," Catalysis Today 89, 233-236.

Timesli, A., Braikat, B., Jamal, M. and Damil, N. [2017] "Prediction of the critical buckling load of multi-walled carbon nanotubes under aial compression," Comptes Rendus Mechanique 345, 158-168.

To, C. W. S. [2006] "Bending and shear moduli of single-walled carbon nanotubes," Finite Elements in Analysis and Design 42, 404-413.

Toomsalu, E., Koppel, I. A. and Burk, P., [2013] "Critical test of some computational chemistry methods for prediction of gas-phase acidities and basicities," Journal of Chemical Theory and Computation 9, 3947-3958.

Tran, N. E. and Lambrakos, S. G. [2005] "Purification and defect elimination of singlewalled carbon nanotubes by the thermal reduction technique," Nanotechnology $\mathbf{1 6}(6), 639$.

Vivien, L., Lancon, P., Riehl, D., Hache, F. and Anglaret, E. [2002] "Carbon nanotubes for optical limiting," Carbon 40, 1789-1797. 
Wang, C. G., Liu, Y. P., Al-Ghalith, J., Dumitrica, T., Wadee M. K. and Tan, H. F. [2016] "Buckling behavior of carbon nanotubes under bending: From ripple to kink," Carbon 102, 224-235.

Wang, C. M., Xiang, Y. and Kitipornchai, S. [2009] "Postbuckling of nano rods/tubes based on nonlocal beam theory," International Journal of Applied Mechanics, 1(02) 259-266.

Ziaee, S. [2014] "Buckling of defective carbon nanotubes under axial and transverse loads," International Journal of Applied Mechanics 6(01), 1450004. 